\title{
Dinámica atmosférica y oceánica en algunos sitios del Área de Conservación Guanacaste (ACG), Costa Rica
}

Omar Gerardo Lizano R. 1,2,3 \& Eric J. Alfaro M. ${ }^{1,2,3}$

1. Centro de Investigación en Ciencias del Mar y Limnología (CIMAR), Universidad de Costa Rica; 11501-2060 San José, Costa Rica;

2. Centro de Investigaciones Geofísicas (CIGEFI), Universidad de Costa Rica, 11501-2060 San José, Costa Rica;

3. Departamento de Física Atmosférica, Oceánica y Planetaria (DFAOP), Escuela de Física, Universidad de Costa Rica, 11501-2060 San José, Costa Rica. omar.lizano@ucr.ac.cr, erick.alfaro@ucr.ac.cr

Recibido 08-X-2013. Corregido 04-III-2014. Aceptado 27-IX-2014.

\begin{abstract}
Atmospheric and oceanic dynamics in some sites of Área de Conservación Guanacaste (ACG), Costa Rica. We studied the oceanographic and meteorological conditions in four sites related to national parks in the Área de Conservación Guanacaste. Variables such as temperature, salinity and dissolved oxygen were studied through the water column in at least one tidal cycle at three bays Tamarindo, Culebra, Santa Elena located in the North Pacific of Costa Rica, and in Ballena Bay, off the Gulf of Nicoya. At the same time, local wind and ocean currents were measured in order to evaluate the dynamic forces at these sites. Environmental variables around Costa Rica were consulted from operational data sources, such as ocean currents and surface wind patterns, to know the prevailing conditions and their influence. During strong trade winds, a typical dry season condition, strong ocean currents are generated in front of the Nicoya Peninsula. This is consistent with the prevailing oceanographic conditions offshore Papagayo Gulf when the wind jet is established. These external forces influence the ocean circulation of open water bays, as was observed in Tamarindo Bay. The local wind and tide are components that modulate local circulation, especially in closed bays like Santa Elena and Ballena. The stratified current structure of the water layers in Ballena Bay showed a typical estuarine condition with the presence of different thermohaline fields dominated by tidal forces. Rev. Biol. Trop. 62 (Suppl. 4): 17-31. Epub 2014 Diciembre 01.
\end{abstract}

Key words: atmospheric dynamic, sea temperature, dissolved oxygen, estuarine circulation, Área de Conservación Guanacaste.

Alfaro, Cortés, Alvarado, Jiménez, Sánchez, Nivia, León \& Ruiz (2012) y Alfaro \& Cortés (2012) elaboraron una caracterización climática de la región costera del Pacifico Norte de Costa Rica, que se resume a continuación. Según estos estudios y de acuerdo a la clasificación del Instituto Meteorológico Nacional de Costa Rica (IMN, http://www.imn.ac.cr, última visita 22/06/2011) esta región tiene un régimen climático que corresponde a la región del Pacífico Norte o Pacífico Seco, ya que presenta un período de disminución de lluvias bastante marcado entre los meses de diciembre y marzo, seguido por dos periodos de máxima precipitación: el primero en mayo-junio y el segundo, mayor que el primero, en agosto-setiembreoctubre. Dichos máximos están separados por un periodo de disminución de la precipitación, normalmente observado en julio, llamado "veranillo" (también conocido como veranillo de San Juan o canícula). Los meses de abril y noviembre se consideran periodos de transición entre las temporadas secas y lluviosas y viceversa (Magaña, Amador \& Medina, 1999; Taylor \& Alfaro, 2005).

Sobre la vertiente del Pacífico de Costa Rica, la magnitud del viento es normalmente más fuerte durante el invierno y primavera boreal, con dirección predominante del este (viento alisio) y disminuye durante el verano y 
otoño boreal (Alfaro, 2002). Durante estas dos últimas estaciones climáticas del año también se observa la formación de sistemas mesoescalares de brisa marina, asociados a aguaceros vespertinos, acompañados algunas veces de tormentas eléctricas. Existen dos sistemas sinópticos transitorios dominantes sobre Costa Rica y que afectan la zona del Pacífico Norte. Durante el invierno-primavera estos son los frentes fríos que incursionan por el Caribe, los cuales refuerzan el viento con componente del este sobre el país. Durante el verano-otoño, la posición más al norte de la Zona de Confluencia Intertropical (ZCIT) intensifica los oestes ecuatoriales (Lizano, 2007).

Propiamente sobre la dinámica de las aguas en costa de Costa Rica varios estudios mencionan la Corriente Costera de Costa Rica (CCCR) (Wyrtki, 1965; Badan-Dangon, 1988; Kessler, 2006; Lavín, Beier, Gómez-Valdés, Godínez \& García, 2006; Brenes, Lavín \& Mascarenhas, 2008). Kessler (2006) también menciona que el flujo hacia el noroeste sobre el lado este del Domo Térmico de Costa Rica, es conocida como la CCCR, con velocidades promedio de cerca de $20 \mathrm{~cm} / \mathrm{s}$, y que continúa bordeando la costa oeste de Centroamérica. Brenes et al. (2008) muestra por primera vez la estructura de esta corriente, y concluye que diferente a la aceptación de un flujo al noroeste, existe un alto grado de variabilidad de esta corriente debido a la presencia de remolinos a lo largo de la costa centroamericana. La dinámica de esta CCCR, y más aún, la interacción que tenga con los rasgos costeros de Costa Rica y su variación estacional, no es conocida. Específicamente sobre el Pacífico Norte de Costa Rica (Golfo de Papagayo), Fonseca (2006) indica que la circulación en esta zona está influenciada por la CCCR que siempre fluye hacia el NW y W, y que además, hay circulaciones locales derivadas de la CCCR según la marea y el oleaje; pero estas aseveraciones no corresponden a mediciones propiamente, sino más bien a inferencias sobre trabajos no publicados.

En este artículo se presentan los resultados de estudios básicos puntuales en algunos sitios del Pacífico Norte de Costa Rica, con el objetivo de identificar las características de algunas variables oceanográficos que apoyaran otros estudios paralelos que se realizaban sobre biología y química marina, y que permitieran ampliar el conocimiento del estado de los hábitats marino-costeros y su conectividad con sitios vecinos. El propósito también es la conservación y manejo de lugares prioritarios del proyecto GRUAS II (Bahía Santa ElenaPunta Gorda-Punta Pargos-Golfo de Papagayo y Cabo Blanco) y las áreas marinas de manejo adyacentes.

\section{MATERIALES Y MÉTODOS}

Se realizó una campaña entre el 29 de febrero y 3 de marzo de 2012, trabajando el 1 de marzo en Bahía Tamarindo, frente al parque marino Las Baulas $\left(10^{\circ} 18^{\prime} 50^{\prime} \mathrm{N}, 8^{\circ} 53^{\prime} 06^{\prime}\right.$ 'W, Fig. 1) y el 2 de marzo en Bahía Culebra $\left(10^{\circ} 35^{\prime} 44^{\prime \prime} \mathrm{N}, 85^{\circ} 42^{\prime} 33^{\prime \prime} \mathrm{W}\right)$. En ambos días se tomaron datos meteorológicos con una estación meteorológica Kestrel 4000, la cual se programó para un intervalo de muestreo de $5 \mathrm{~min}$.

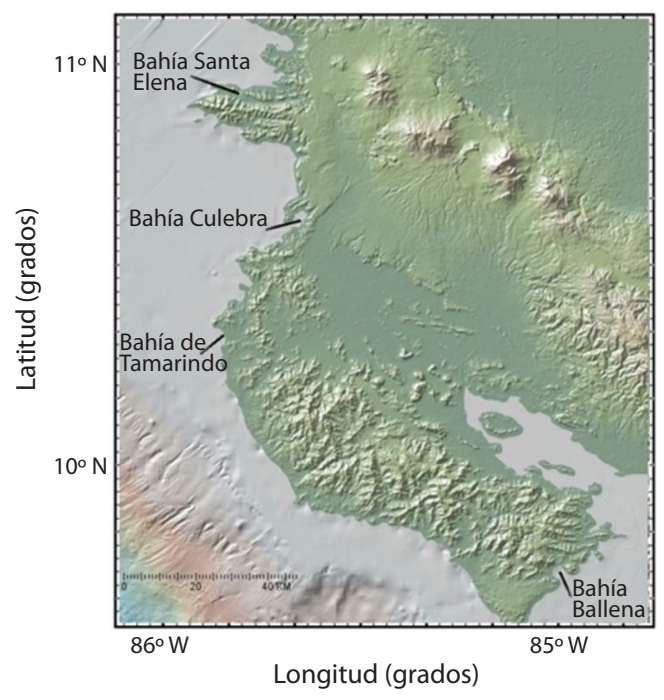

Fig. 1. Ubicación de estaciones de muestreo en Bahía Santa Elena, Bahía Culebra, Bahía Tamarindo y Bahía Ballena. Imagen editada de GeoMapApp ver. 3.3.8.

Fig. 1. Location of the simple sites in Santa Elena, Culebra Tamarindo and Ballena bay. Edited image from GeoMapApp ver. 3.3.8. 
Se realizaron perfiles de CTD el 1 de marzo frente al parque marino Las Baulas, a aproximadamente $30 \mathrm{~m}$ de profundidad $\left(10^{\circ} 18^{\prime} 50^{\prime} \mathrm{N}, 85^{\circ} 53^{\prime} 06^{\prime \prime} \mathrm{W}\right)$. Mediciones de las corrientes marinas en la columna de agua se realizaron con un ADCP (Acustic Doppler Current Profiler). Se utilizaron 24 celdas de aproximadamente $2 \mathrm{~m}$ para medir las corrientes cada 5min a través de la columna de agua. El 2 de marzo se realizaron las mismas mediciones al frente de la Bahía Culebra $\left(10^{\circ} 35^{\prime} 44^{\prime \prime} \mathrm{N}\right.$, $85^{\circ} 42^{\prime} 33^{\prime \prime} \mathrm{W}$ ).

La segunda campaña se realizó al sector de la Bahía Santa Elena $\left(10^{\circ} 56^{\prime} 35^{\prime \prime} \mathrm{N}\right.$, $\left.85^{\circ} 48^{\prime} 16^{\prime \prime} \mathrm{W}\right)$, en junio de 2012 . Se utilizaron dos estaciones meteorológicas portátiles Kestrel tipo $4500 \mathrm{NV}$, las cuales se ubicaron una expuesta y otra a la sombra. Las variables que se midieron con estas estaciones fueron: Temperatura Superficial del Aire, Humedad Relativa, Magnitud de la Velocidad del Viento, Dirección de la Velocidad del Viento y Presión Superficial. Las mediciones de las estaciones automáticas se realizaron el 20 de junio cada $5 \mathrm{~min}$. Las mediciones de CTD se realizaron en la boca de la Bahía Santa Elena (8448'16" W, $\left.10^{\circ} 56^{\prime} 36^{\prime \prime} \mathrm{N}\right)$ el 20 de junio del 2012. Mediciones de corrientes (ADCP) también se realizaron durante ese período. El 31 de julio del 2012 se visitó Bahía Ballena, en el Golfo de Nicoya. Mediciones de CTD se realizaron en la boca de la bahía $\left(84^{\circ} 58^{\prime} 46^{\prime \prime} \mathrm{W}, 9^{\circ} 42^{\prime} 51^{\prime}\right.$ 'N), comenzando a las 8:10 am y terminando a las 4:24 pm.

La batimetría de la mayoría de estas bahías se realizaron digitalizando contornos batimétricos, de al menos cada 2 metros, de las cartas náuticas BlueChart G2 Vision de Garmin. Modelos de Elevación Digital (MED) se elaboraron con interpolaciones de aproximadamente $30 \mathrm{~m}$ con el paquete SURFER ver. 9.0

Para identificar las condiciones oceanográficas mar adentro y comparar con las condiciones locales, se recopilaron datos oceanográficos de bases de datos internacionales, archivados en el Programa MIO (Módulo de Información Oceanográfica, CIMAR, www. miocimar.ucr.ac.cr) como mareas y corrientes marinas. Datos de viento se obtuvieron de los reanálisis de NCAR (National Center for Atmospheric Research) de la NOAA (National Oceanic and Atmospheric Administration).

\section{RESULTADOS}

\section{A. Condiciones océano-meteorológicas en Bahía Tamarindo y en Bahía Culebra en marzo del 2012.}

Condiciones meteorológicas locales. No se registró precipitación durante los muestreos del 1 de marzo, observándose las 8 octas del cielo sin cobertura nubosa. Al analizar los datos, sin embargo se encontró que no todos los datos del anemómetro de la estación fueron registrados, por alguna causa que se desconoce. Debido a lo anterior, se solicitaron los datos de dos estaciones meteorológicas, pertenecientes al Instituto Meteorológico Nacional, cercanas a los sitios de muestreo identificadas como Liberia $\left(74051,10^{\circ} 35^{\prime} 36^{\prime \prime} \mathrm{N}, 85^{\circ} 32^{\prime} 40^{\prime \prime} \mathrm{W}\right)$ y Pinilla (72149, $\left.10^{\circ} 15^{\prime} 10^{\prime \prime} \mathrm{N}, 85^{\circ} 50^{\prime} 34^{\prime \prime} \mathrm{W}\right)$, con una resolución temporal horaria.

Durante el día 1, se observó in situ, una rapidez promedio del viento de $8,1 \mathrm{~m} / \mathrm{s}$ con una desviación estándar de $1,4 \mathrm{~m} / \mathrm{s}$ y un rango de 5,9 a $9,9 \mathrm{~m} / \mathrm{s}$, con una dirección predominante del Noreste durante todo el día. Para el día 2, las características del viento durante la mañana fueron similares a las observadas durante el día anterior con una rapidez promedio del viento de $7,3 \mathrm{~m} / \mathrm{s}$ con una desviación estándar de $2,0 \mathrm{~m} / \mathrm{s}$ y un rango de 3,7 a $9,6 \mathrm{~m} / \mathrm{s}$, con una dirección predominante del Noreste, sin embargo la magnitud del viento se debilitó durante las horas de la tarde registrándose una rapidez promedio del viento de $2,4 \mathrm{~m} / \mathrm{s}$ con una desviación estándar de $1,6 \mathrm{~m} / \mathrm{s}$ y un rango de viento calmo hasta $4,2 \mathrm{~m} / \mathrm{s}$, con una dirección predominante del Nornoroeste.

Dicho comportamiento también fue observado en las estaciones de Liberia y Pinilla (Fig. 2). Durante el día 1 las estaciones mostraron una rapidez promedio del viento de 10,2 y $9,7 \mathrm{~m} / \mathrm{s}$, respectivamente, con una desviación estándar en ambas de $0,9 \mathrm{~m} / \mathrm{s}$, con una dirección 


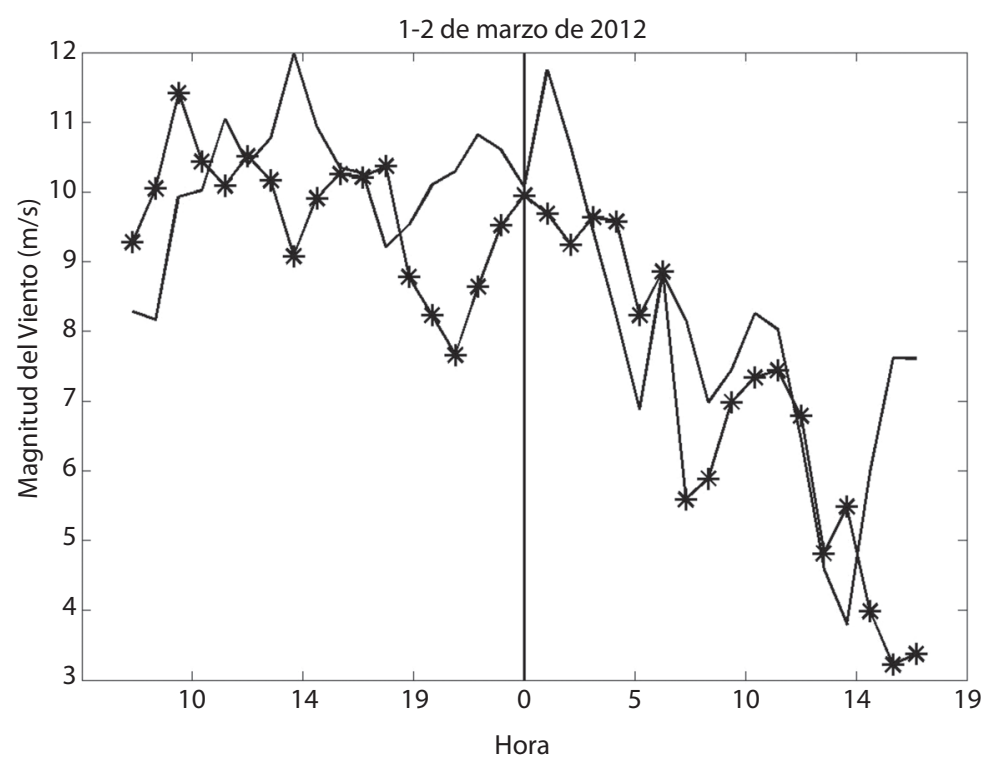

Fig. 2. Rapidez del viento observada en las estaciones de Liberia (línea continua) y Pinilla (línea con asteriscos).

Fig. 2. Wind speed observed in the stations of Liberia (solid line) and Pinilla (line with asterisks).

predominante del Este-Noreste. Nótese además como fue disminuyendo la magnitud del viento conforme avanzó el día 2. Cabe destacar que en ambas estaciones durante ese día la dirección predominante también fue del Este-Noreste, excepto para las horas de la tarde en Pinilla en donde se observó del oeste.

\section{Condiciones oceanográficas en Bahía} Tamarindo. La Fig. 3 muestra la variación en el tiempo de los perfiles realizados en la columna de agua del CTD que se hicieron en Bahía Tamarindo (frente al Parque Nacional Las Baulas).

Es notorio en gráficas anteriores, el hundimiento de los contornos de temperatura, salinidad y oxígeno, entre las 9 y 10 am, justo cuando el viento estuvo soplando más fuerte sobre la región, y justo después de la marea alta (marcada en la Fig. 3A).

Las corrientes marinas a lo largo de la columna de agua también fueron muy intensas durante la mañana, especialmente en la superficie, como lo muestra la parte inferior de la Fig. 4A. Magnitudes de hasta $83 \mathrm{~cm} / \mathrm{s}$ fueron medidas durante el período de la mañana (Fig. 4A). Su dirección fue permanentemente hacia el norte (como lo muestran los vectores en la parte superior de la Fig. 4A), solamente variando su magnitud a través del tiempo. Las corrientes débiles se dieron en la tarde, y volvieron a ser ligeramente más intensa al final del período de medición, después de la marea baja.

Condiciones oceanográficas locales frente a Bahía Culebra: Bahía Culebra es una bahía semi-cerrada, que está en el interior del Golfo de Papagayo. Se hace más profunda hacia su boca, donde alcanza un máximo de 40-45m (Fig. 5).

En cuanto a los perfiles de CTD, condiciones más estables y menos variables se midieron de la temperatura, salinidad y oxígeno disuelto (Fig. 6) frente a esta bahía el día siguiente ( 2 de marzo), y esto fue más evidente justo cuando el viento se hizo más débil, como mostraba la Fig. 2.

En esta última, es de resaltar la mayor cantidad de oxígeno disuelto en la capa superficial, por encima de los $10 \mathrm{~m}$ de profundidad. 

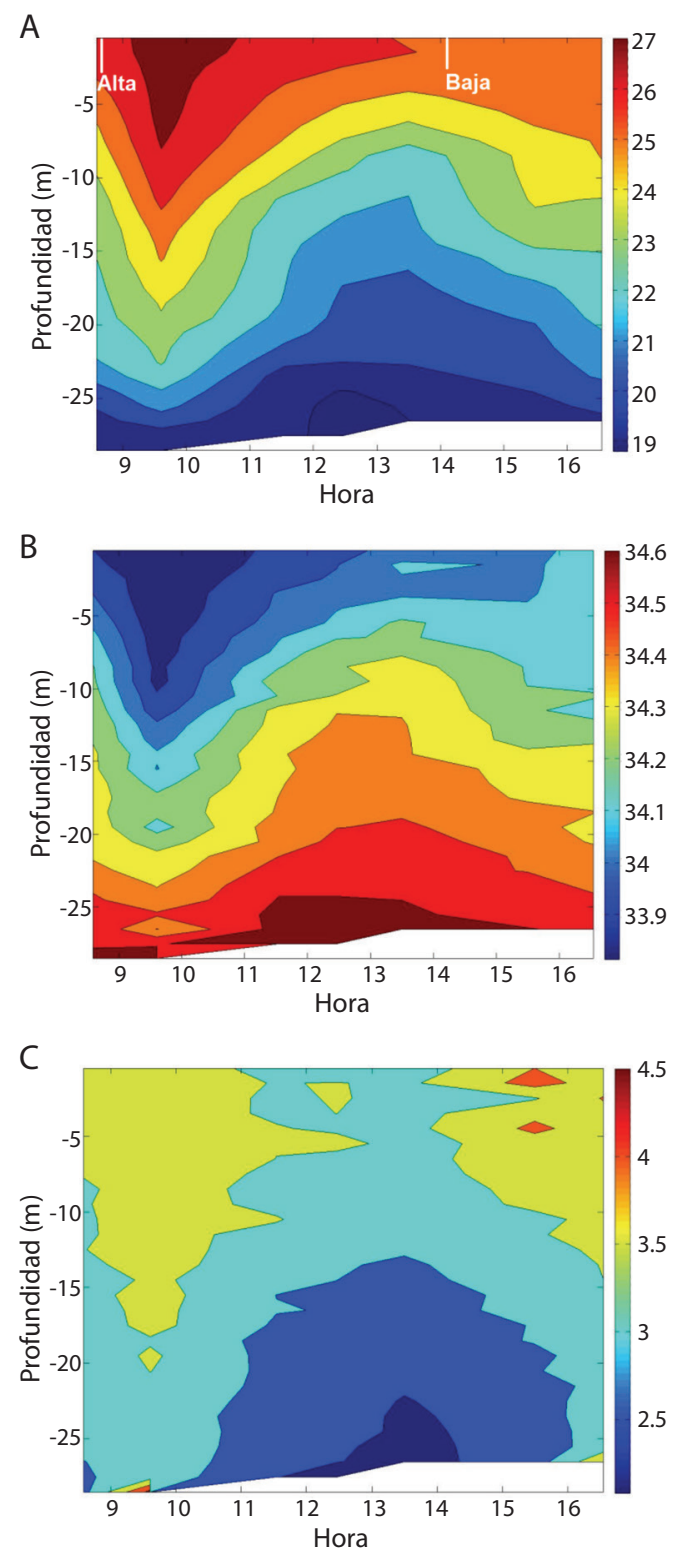

Fig. 3. Variación en el tiempo de los perfiles de A: temperatura $\left({ }^{\circ} \mathrm{C}\right), \mathrm{B}$ : salinidad (UPS) y C: oxígeno disuelto (mg/l) en Bahía Tamarindo.

Fig. 3. Variation in time of the profiles of A: temperature $\left({ }^{\circ} \mathrm{C}\right)$, B: salinity (PSU) and $\mathrm{C}$ : dissolved oxygen $(\mathrm{mg} / \mathrm{l})$ observed in Tamarindo Bay.

Las corrientes marinas, como muestra la Fig. 7, fueron menos intensas este día. Velocidades máximas de $35 \mathrm{~cm} / \mathrm{s}$ en la mañana (Fig. 7A) y de $37 \mathrm{~cm} / \mathrm{s}$ en la tarde (Fig. 7B) fueron
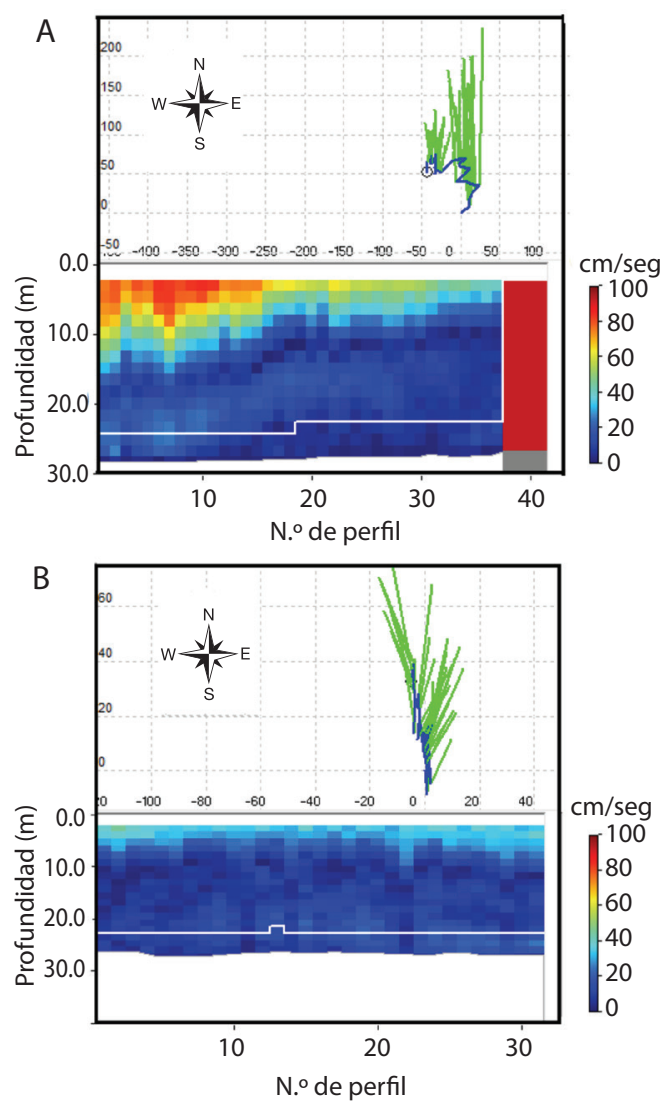

Fig. 4. Magnitud y dirección de las corrientes marinas promedio integrada en la columna de agua en Bahía Tamarindo el 1 de marzo en A: de 9:02 am a 12:43 pm y B: $12: 45 \mathrm{~m}$ a $3: 15 \mathrm{pm}$.

Fig. 4. Marine current magnitude and direction integrated in the water column in Tamarindo Bay, March 1, from 9:02 am to $12: 43 \mathrm{pm}(\mathrm{A})$ and from $12: 45 \mathrm{pm}$ to $3: 15 \mathrm{pm}(\mathrm{B})$.

medidas. Su dirección fue permanentemente hacia el sur, como indican los vectores en la parte superior de las Fig. 7A y 7B. La Fig. 7A muestra una estratificación en el flujo: una capa con mayor velocidad a aproximadamente $30 \mathrm{~m}$.

\section{B. Condiciones meteorológicas y oceano- gráficas en Bahía Santa Elena el 20 de junio del 2012.}

Condiciones meteorológicas locales. Durante ese día se observó oleaje de fondo con viento de dirección predominante del sureste, aumentando su magnitud conforme fue 


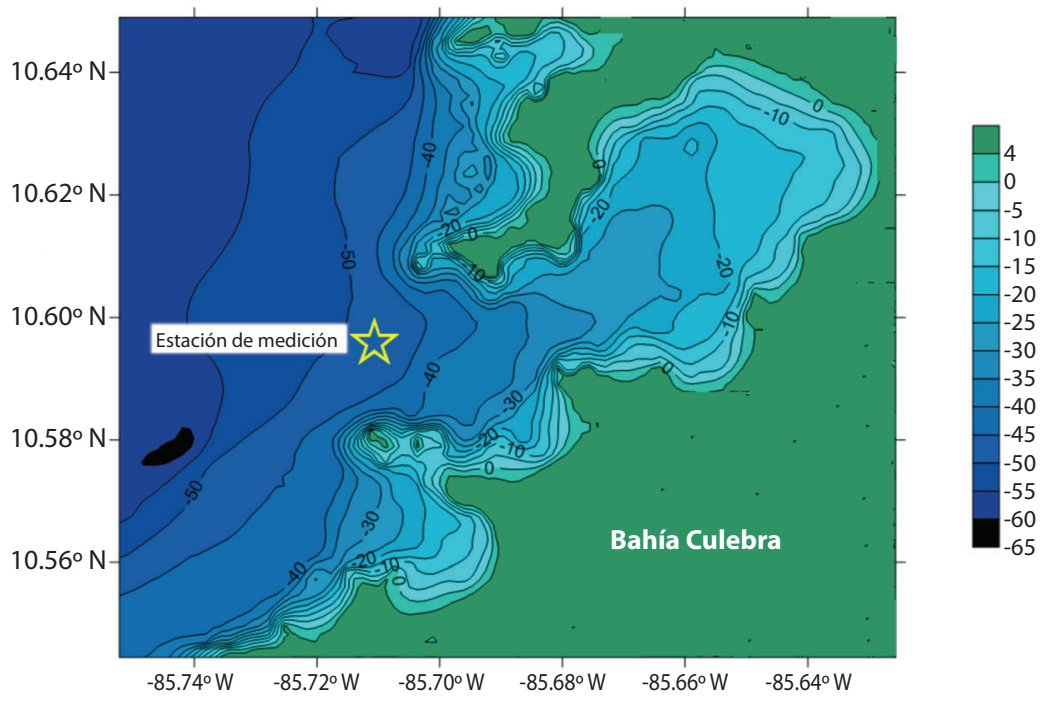

Fig. 5. Contornos batimétricos (m) en Bahía Culebra.

Fig. 5. Batimetric contours ( $\mathrm{m}$ ) in Culebra Bay. The star shows the location of the station.

avanzando la hora del día. Presentó una rapidez promedio del viento de $3,0 \mathrm{~m} / \mathrm{s}$ con una desviación estándar de $1,5 \mathrm{~m} / \mathrm{s}$ y un rango de 0 a $5,8 \mathrm{~m} / \mathrm{s}$ (Fig. 8). Se observaron nubes bajas cumuliformes sobre tierra y cirrus al norte, para un estimado de aproximadamente 6 octas, sin que se registrara precipitación.

\section{Condiciones oceanográficas en Bahía} Santa Elena el 20 de junio del 2012. Bahía Santa Elena es una bahía semi-cerrada que está dentro del Golfo de Santa Elena. Su circulación debería ser muy restringida dada su posición. La Fig. 9 muestra una mayor resolución de la batimetría de esta bahía. Profundidades de 30-35m se indican en la boca de la misma. Hacia el interior es relativamente somera.

La Fig. 10 indica la variación en el tiempo de los perfiles de algunas variables oceanográficas en el sitio de muestreo (Fig. 9) en la boca de la Bahía Santa Elena. En este sitio, la columna de agua es menos estratificada respecto a la de Bahía Tamarindo y Bahía Culebra. Valores muy bajos de oxígeno (valor máximo: $0,55 \mathrm{mg} / \mathrm{l}$ ) se midieron durante todo el día frente a Santa Elena, lo cual podría ser un indicativo de la poca circulación en la bahía.
La Fig. 11 muestra la variación de las corrientes marinas frente a la Bahía de Santa Elena. En la Fig. 11A, se nota la estratificación de velocidades en la mañana, siendo esta velocidad con direcciones hacia afuera de la bahía durante la marea bajando (ver Fig. 9). En la tarde, el flujo de corrientes cambió de dirección, siendo hacia adentro de la bahía (hacia el sureste, Fig. 11B superior), y justo cuando la marea comenzó a subir. Las velocidades se incrementaron en este período (Fig. 11B), así como también las velocidades del viento (Fig. 8).

\section{Condiciones meteorológicas y oceano- gráficas en Bahía Ballena el 31 de julio del 2012.}

Condición meteorológica local. Durante ese día se observó oleaje de fondo y hacia el medio día el mar de viento predominó, volviendo a dominar el mar de fondo conforme avanzó la tarde. La dirección predominante del viento fue del sureste disminuyendo hacia el final del periodo de medición. Presentó una rapidez promedio del viento de $9,2 \mathrm{~m} / \mathrm{s}$ con una desviación estándar de $2,3 \mathrm{~m} / \mathrm{s}$ y un rango de 0 a $8,3 \mathrm{~m} / \mathrm{s}$ 

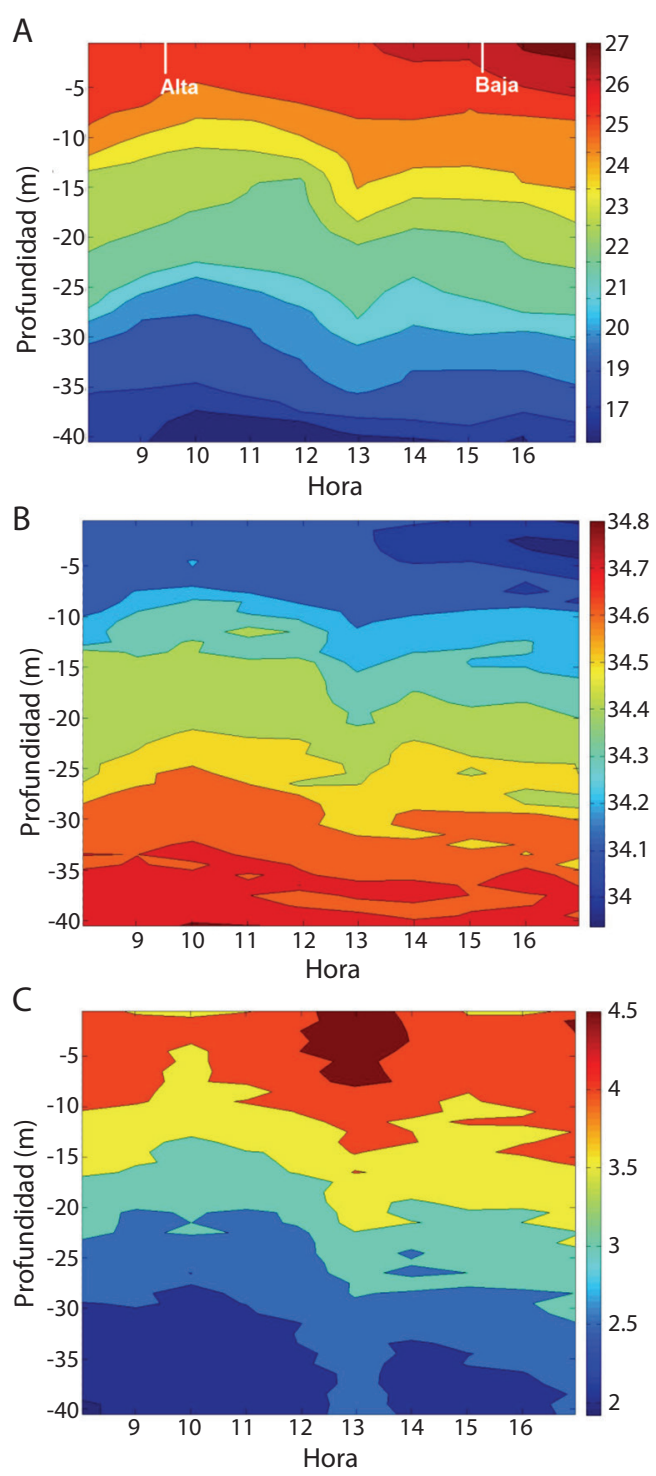

Fig. 6. Variación en el tiempo de los perfiles de A: temperatura $\left({ }^{\circ} \mathrm{C}\right)$, B: salinidad (UPS) y C: oxígeno disuelto $(\mathrm{mg} / \mathrm{l})$ frente a Bahía Culebra.

Fig. 6. Variation in time of the profiles of A: temperature $\left({ }^{\circ} \mathrm{C}\right)$, B: salinity (PSU) and $\mathrm{C}$ : dissolved oxygen $(\mathrm{mg} / \mathrm{l})$ observed in Culebra Bay.

(Fig. 12). El estimado de la nubosidad fue de 6 a 8 octas, de tipo cumuliforme, sin que se registrara precipitación en el sitio de medición.
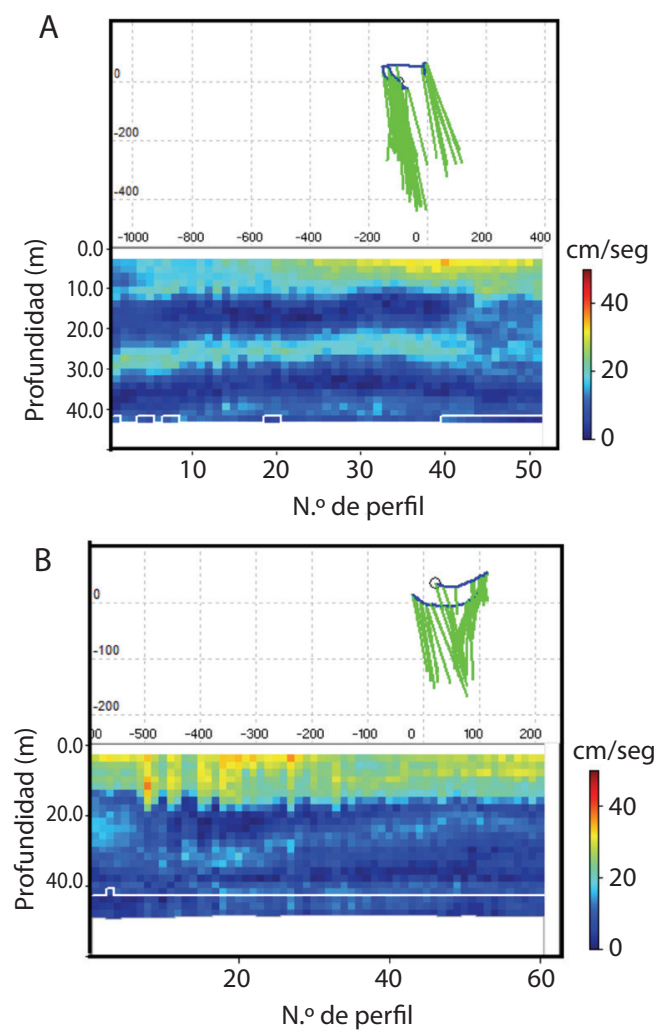

Fig. 7. Magnitud y dirección de las corrientes marinas en la columna de agua frente a Bahía Culebra el 02 de marzo en A: de 7:39 am a 11:49 am y B: 12:05 pm a 5:00 pm.

Fig. 7. Marine current magnitude and direction integrated in the water column in Culebra Bay, March 02, in A: from 7:39 am to $11: 49$ am and $B$ : from 12:05 pm to $5: 00 \mathrm{pm}$.

Condiciones oceanográficas en Bahía Ballena, Guanacaste, el 31 de julio del 2012. Bahía Ballena es una bahía (Fig. 13) que está en la parte externa del Golfo de Nicoya (Fig. 1). Esta región tiene una mayor dinámica de corrientes que la parte interna del Golfo (Lizano \& Alfaro, 2004) e influenciada por el oleaje desde mar adentro (Lizano, 2007).

Como indica la figura anterior, Bahía Ballena es una bahía somera, con profundidades máximas de 30-35m en la parte externa.

La Fig. 14 muestra la variación en el tiempo de los perfiles de algunas variables 


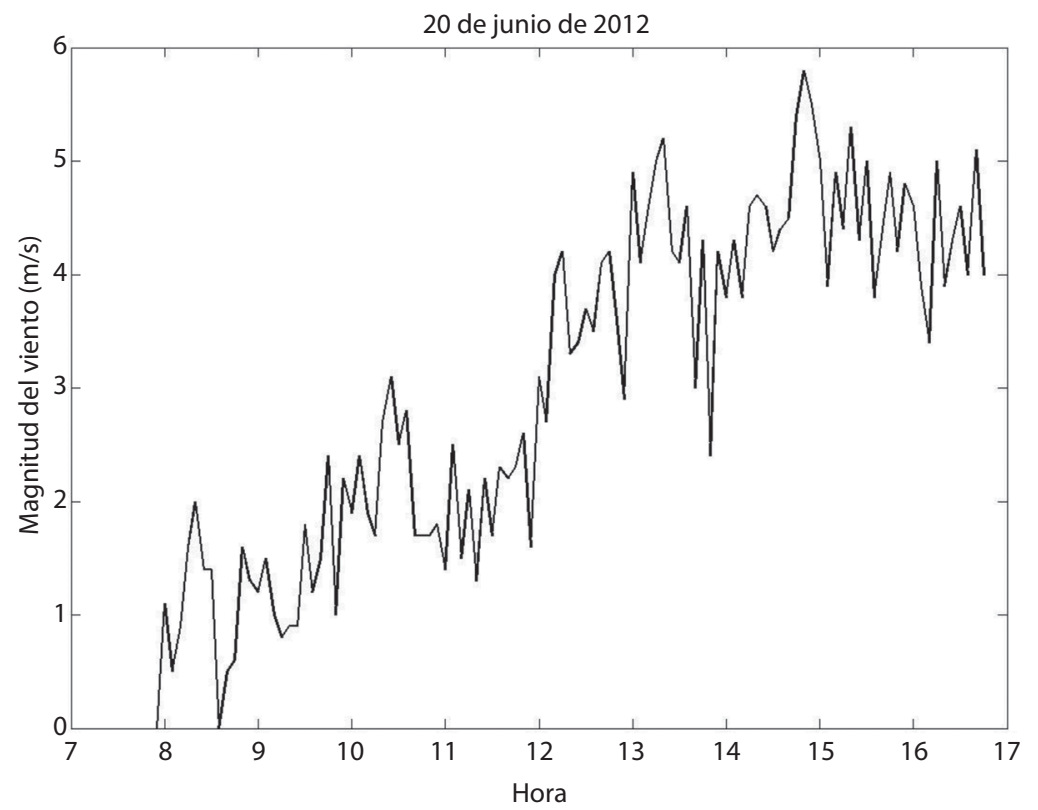

Fig. 8. Rapidez del viento observada en Bahía Santa Elena. Fig. 8. Wind speed observed in Santa Elena Bay.

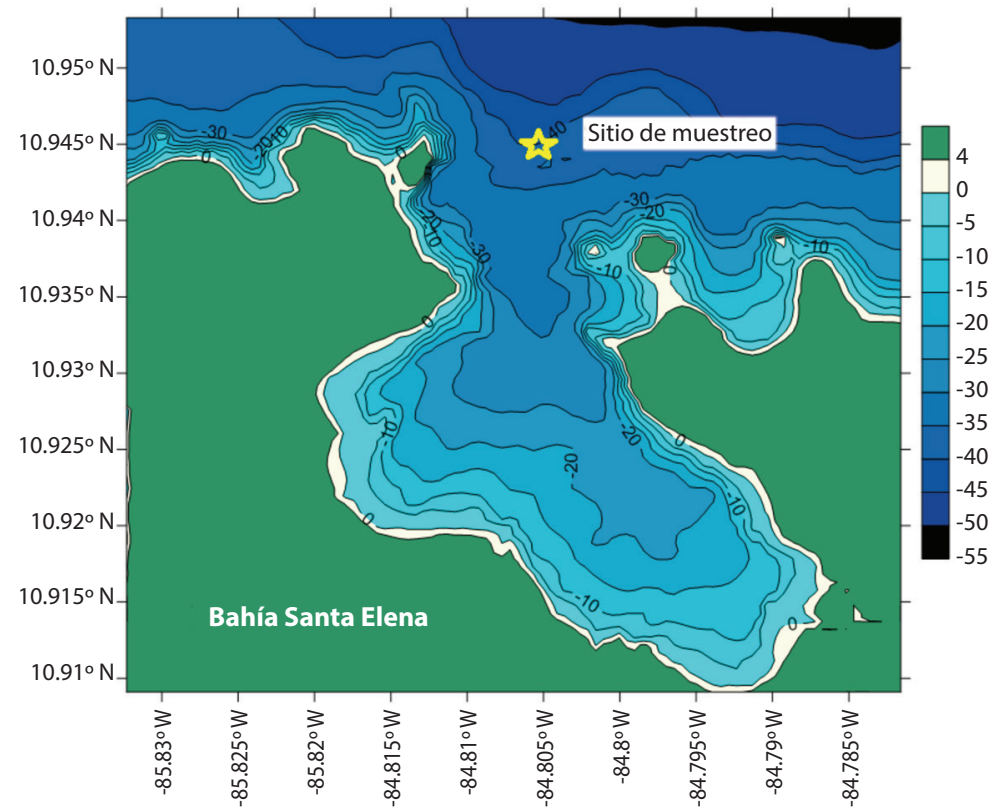

Fig. 9. Contornos batimétricos $(\mathrm{m})$ de Bahía Santa Elena.

Fig. 9. Batimetric contours (m) in Santa Elena Bay. The star shows the location of the station. 

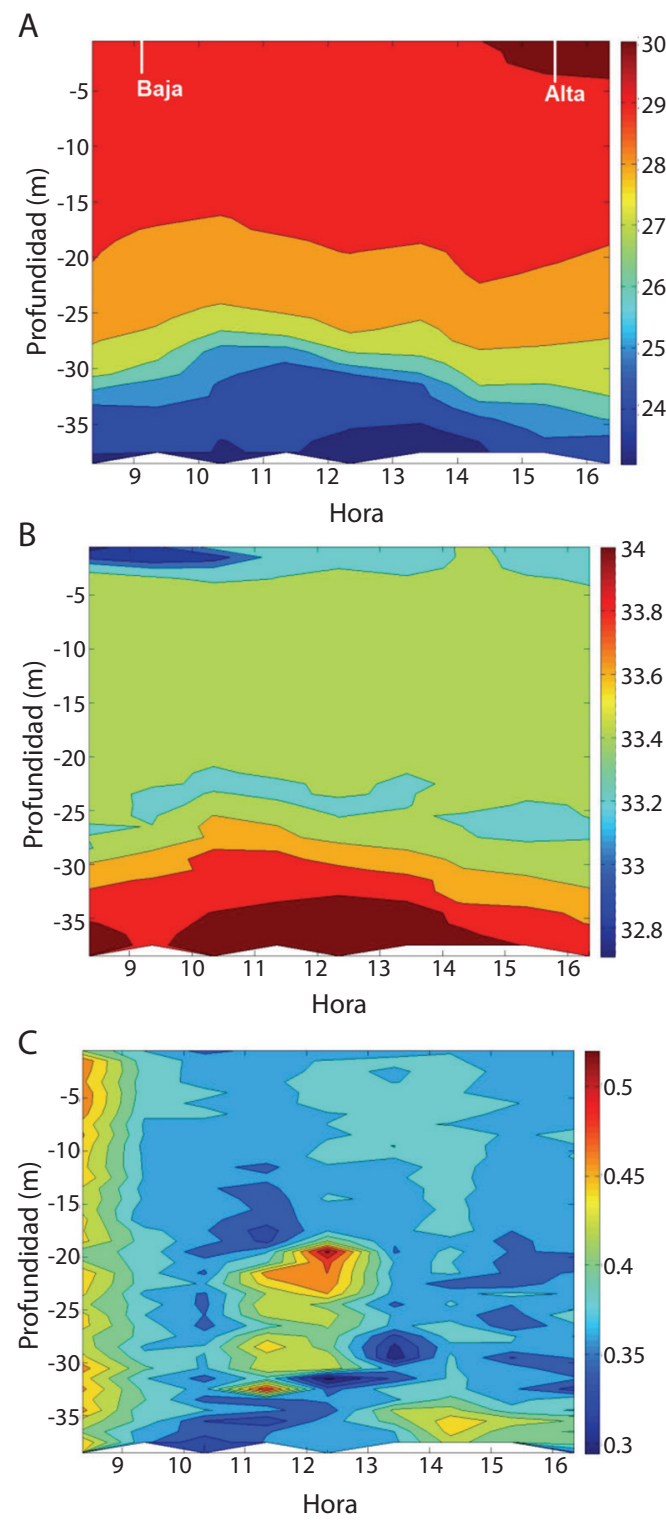

Figs. 10. Variación en el tiempo de los perfiles de A: temperatura $\left({ }^{\circ} \mathrm{C}\right), \mathrm{B}$ : salinidad (UPS) y C: oxígeno $(\mathrm{mg} / \mathrm{l})$ disuelto frente a Bahía Santa Elena.

Fig. 10. Variation in time of the profiles of A: temperature $\left({ }^{\circ} \mathrm{C}\right)$, B: salinity (PSU) and $\mathrm{C}$ : dissolved oxygen $(\mathrm{mg} / \mathrm{l})$ observed in Santa Elena Bay.

oceanográficas frente a Bahía Ballena. Es clara la intrusión de aguas más frías, salinas y de menor cantidad de oxígeno a partir de las 11 de la mañana, cuando la marea iba subiendo (marea baja en Puntarenas a las 6:39 hrs). En
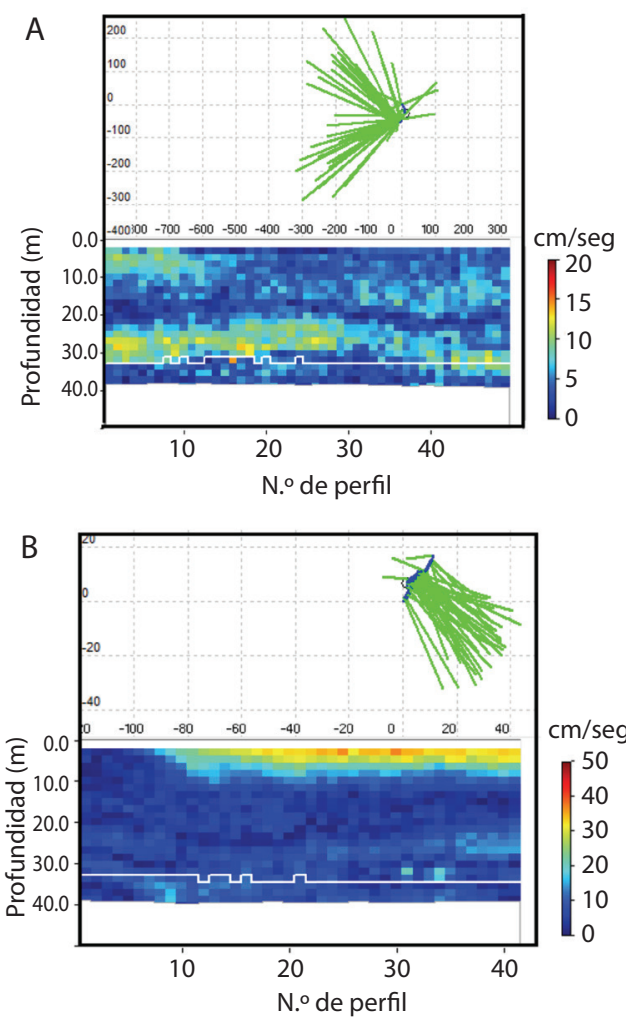

Fig. 11. Magnitud y dirección de las corrientes marinas en la columna de agua frente a Bahía Santa Elena el 20 de junio en A: entre 8:30 am y 12:30 pm y B: entre 1:03 pm y $4: 23 \mathrm{pm}$.

Fig. 11. Marine current magnitude and direction integrated in the water column in Santa Elena Bay, June 20, in A: from 8:30 am to $12: 30 \mathrm{pm}$ and B: from 1:03 pm to 4:23 pm.

este tiempo también el viento disminuyó su intensidad. Es clara la estratificación de salinidad a través de la columna de agua, típica de este estuario (Voorhis, Epifanio, Don Maurer, Dittel \& Vargas, 1983). La capa superficial uniforme de salinidad se hace más profunda alrededor de la marea baja, y se va haciendo más delgada conforme la marea sube. Un cambio en la pendiente de las curvas de salinidad y temperatura se logra distinguir alrededor de las 3:00 pm, para continuar con el ciclo hacia la próxima marea baja. Mayor cantidad de oxígeno disuelto se nota en la columna de agua en las primeras horas de la mañana, y alrededor de la marea baja. En este sitio se encontró la mayor 


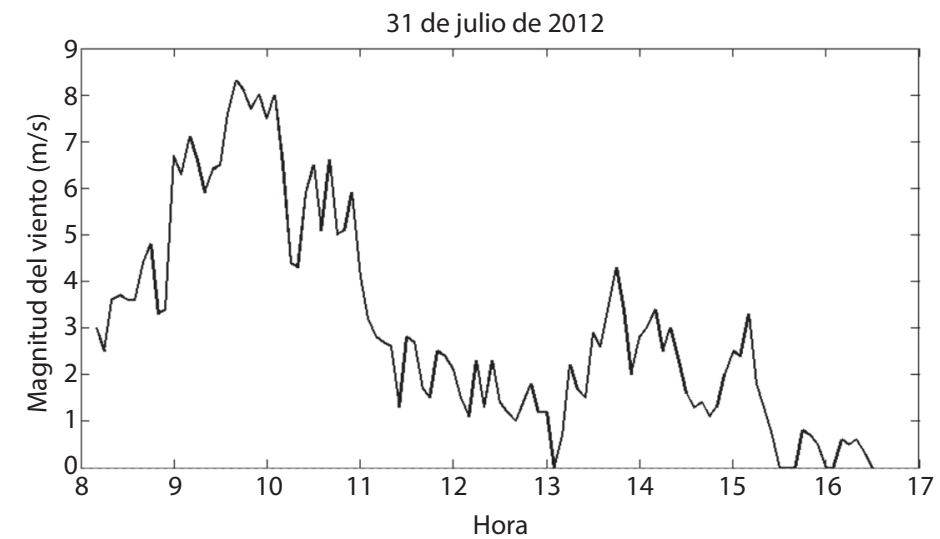

Fig. 12. Rapidez del viento observada en Bahía Ballena.

Fig. 12. Wind speed observed in Ballena Bay.

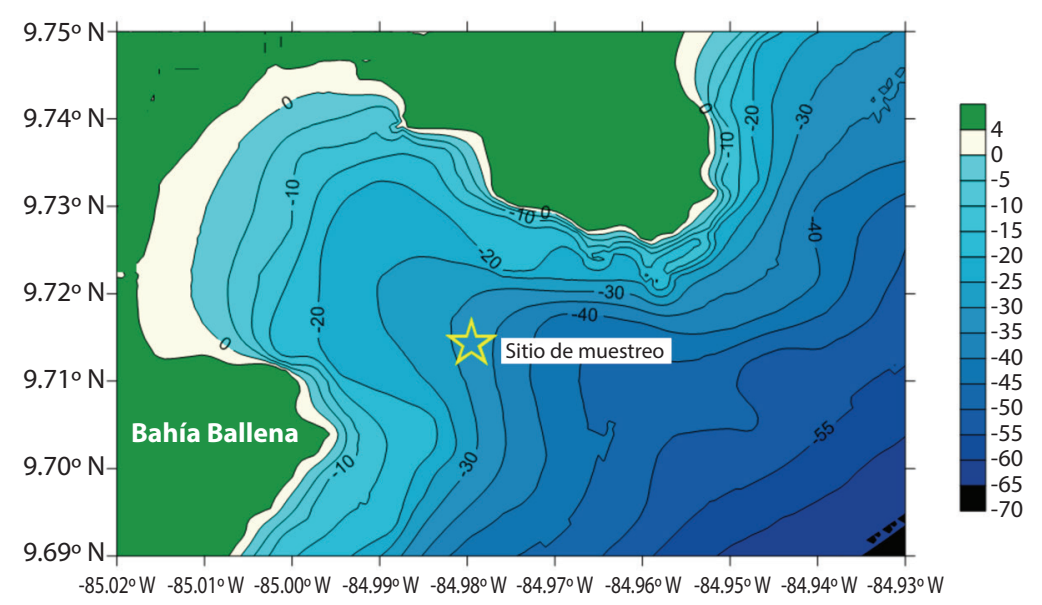

Fig. 13. Contornos batimétricos $(\mathrm{m})$ en Bahía Ballena.

Fig. 13. Batimetric contours (m) in Ballena Bay. The star shows the location of the station.

cantidad de oxígeno $(5,5 \mathrm{mg} / \mathrm{l})$ respecto de los otros sitios muestreados.

La Fig. 15 muestra las características de las corrientes marinas en la columna de agua de este sitio. Estas corrientes se orientan según el ciclo de marea. Mientras la marea sube en Puntarenas (marea alta a las 13:00 hrs, ver Fig. 14A), frente a Bahía Ballena el agua se dirige hacia el noroeste y oeste, ingresando hacia el interior, y cuando la marea está bajando en Puntarenas, el flujo comienza a cambiar hacia el sur y luego sureste, saliendo de la bahía. Los vectores de la Fig. 15A no pudieron interpretarse por un fallo en el programa de extracción de información del ADCP.

En ambas figuras anteriores se nota una estratificación de flujo en la columna de agua, siendo el flujo por el fondo de mayor intensidad en ambos casos. Velocidades máximas de $40 \mathrm{~cm} / \mathrm{s}$ muestra la Fig. $15 \mathrm{~A}$ en el fondo, y de $31 \mathrm{~cm} / \mathrm{s}$ en el fondo en la Fig. 15B. En superficie se registraron $28 \mathrm{~cm} / \mathrm{s}$ y $24 \mathrm{~cm} / \mathrm{s}$ respectivamente. Pero además, se estableció una estratificación en dirección de la corriente en 

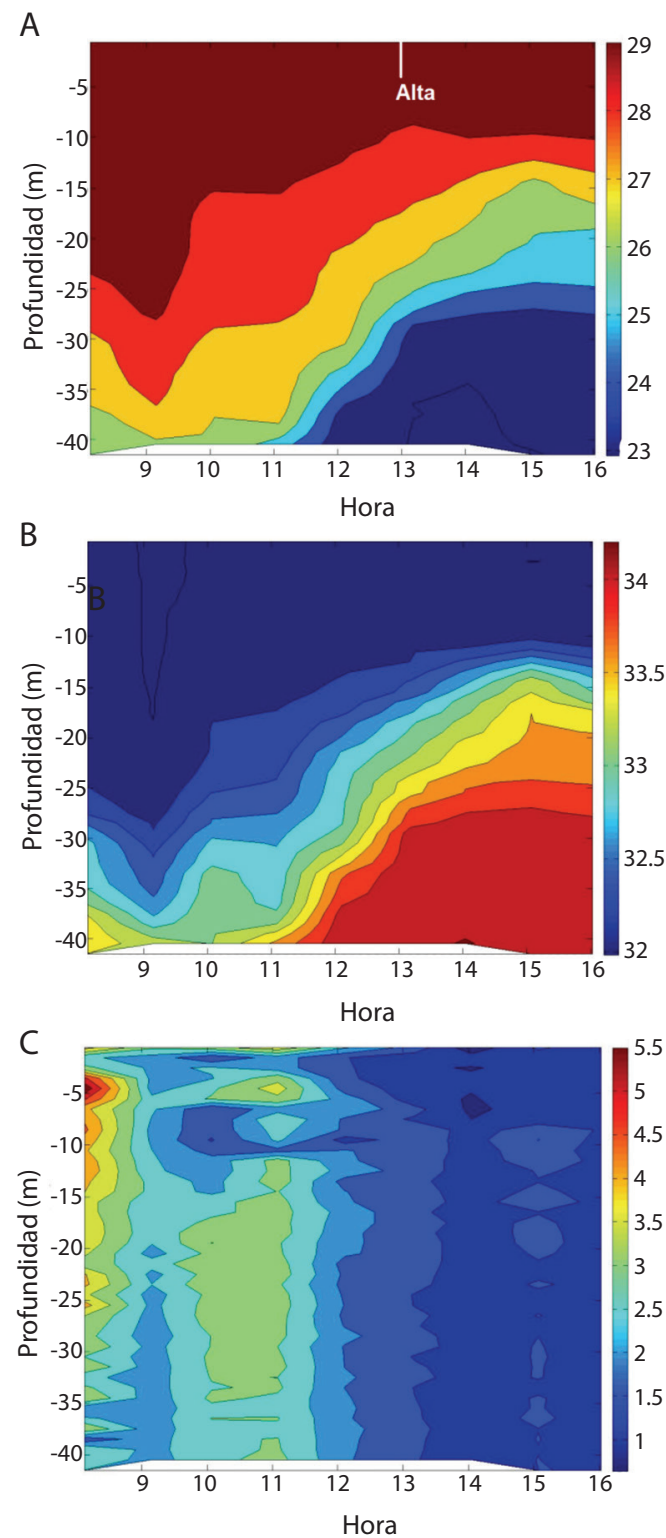

Figs. 14. Variación en el tiempo de los perfiles de A: salinidad (UPS), B. temperatura $\left({ }^{\circ} \mathrm{C}\right)$ y oxígeno disuelto (mg/l) frente a Bahía Ballena el 31 de julio.

Fig. 14. Variation in time of the profiles of A: temperature $\left({ }^{\circ} \mathrm{C}\right)$, B: salinity (PSU) and C. dissolved oxygen $(\mathrm{mg} / \mathrm{l})$ observed in Ballena Bay, july 31.

la columna de agua, donde en la capa sobre los $20 \mathrm{~m}$, el agua salía de la bahía, y bajo los 20m, el agua entraba en la bahía, un intercambio de agua esperado en una bahía semi-cerrada.
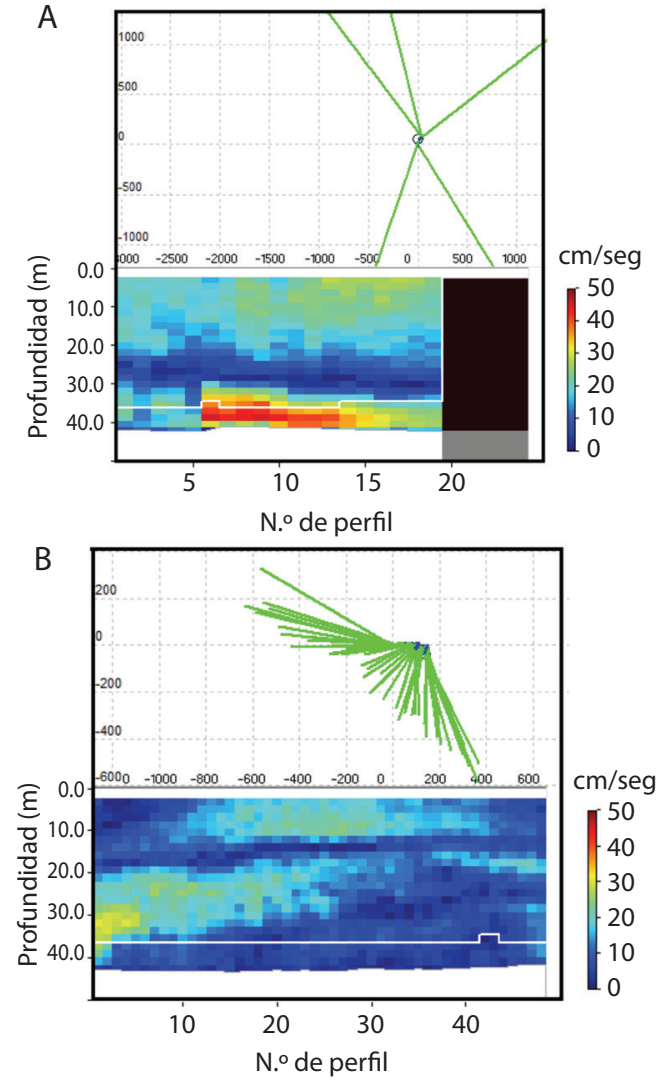

Fig. 15. Magnitud y dirección de las corrientes marinas en la columna de agua frente a Bahía Ballena el 31 de julio en A: de 8:30 am a 12:30 pm, y B: de 1:03 pm a 4:23 pm. Fig. 15. Marine current magnitude and direction integrated in the water column in Ballena Bay, July 31, A: from 8:30 am to $12: 30 \mathrm{pm}$ and $\mathrm{B}$ : from 1:03 pm to $4: 23 \mathrm{pm}$.

\section{DISCUSIÓN}

\section{Condiciones regionales y locales para} la campaña de marzo y junio del 2012. La consulta en base de datos de los resultados de modelos océano-meteorológicos mostraban que en marzo las condiciones atmosféricas en el Pacífico Norte de Costa Rica, correspondían con la época seca (Alfaro et al., 2012; Alfaro \& Cortés, 2012). Esto se pudo comprobar en la campaña a Bahía Tamarindo y a Bahía Culebra (1 y 2 de marzo), donde no se registró ninguna precipitación y hubo cielo completamente despejado. El viento durante esos días 
(Fig. 16A) era el típico viento alisio fuerte que se proyecta desde el Caribe hacia el Pacífico Norte, formando un chorro de viento (Amador et al., 2006) al frente del Golfo de Santa Elena. Las mediciones que se rescataron de Liberia y de Pinilla (Fig. 2), fueron coherentes con estas condiciones, pues se alcanzaron máximos de hasta $12 \mathrm{~m} / \mathrm{s}$. Mientras que en contraste, durante el muestreo de junio, prevalecieron los vientos oestes (Fig. 16B), con velocidades más bien débiles. Las mediciones que se hicieran en la boca de Santa Elena (Fig. 8B), indicaron velocidades promedio de $3.0 \mathrm{~m} / \mathrm{s}$ y con dirección hacia el noroeste (viento sureste), posiblemente por influencia topográfica local.

Una revisión de bases de datos los resultados de modelos numéricos sobre las corrientes marinas regionales prevalecientes alrededor de marzo, se muestran en la Fig. 17. Las corrientes fueron intensas frente a la Península de Nicoya el 01 de marzo del 2012, y con una componente fuerte hacia el norte-noroeste, como lo muestra la Fig. 17A. Es común para esta época que se genere una corriente fuerte hacia el noreste frente a la Península de Nicoya, donde el viento y la temperatura superficial del mar, obliga a una circulación anticiclónica (contra las manecillas del reloj), como es reportado por varios estudios anteriores sobre la dinámica atmosférica y oceanográfica en esta región (Amador et al., 2006; Kessler, 2006; Fiedler, 2002). Esta corriente permanente y fuerte para esta época, tiene gran importancia para organismos de algunas de las playas de esta región de Pacífico Norte, que muy probablemente utilizan esta dinámica para alejarse o regresar a la costa.

La Bahía Tamarindo al ser una bahía abierta, está más expuesta a esta gran circulación oceánica, por lo que muestra un patrón permanente de corrientes hacia el norte (Fig. 4). Sólo se hace más débil alrededor de la marea baja (marea baja a las 14:18 hrs). Se debe resaltar que los vientos tuvieron una componente noreste durante esos días, sin embargo, las corrientes marinas en el sitio, se mantuvieron con una componente norte todo el tiempo, indicando esto el dominio de la dinámica de
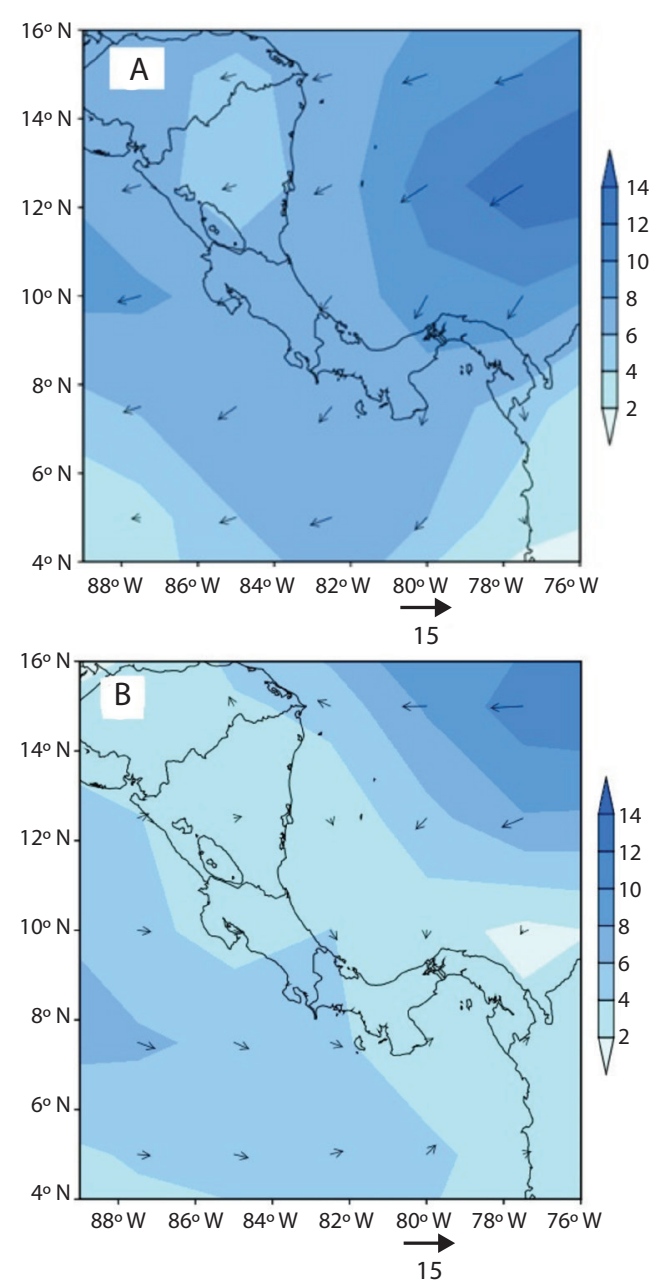

Figs. 16. Patrón del vientos frente a la Península de Nicoya el A: 1 de marzo y el B: 20 de junio. Reanálisis de viento de National Centers for Environmental Prediction (NCEP) and the National Center for Atmospheric Research (NCAR).

Figs. 16. Wind pattern off the Nicoya Peninsula, A: March 1 and B: June 20. From wind Reanalysis, National Centers for Environmental Prediction (NCEP) and the National Center for Atmospheric Research (NCAR).

las corrientes externas en este sitio. Muy poca influencia se notó debido al cambio de mareas.

Bajo condiciones ambientales similares, en Bahía Culebra el flujo de corrientes fue hacia el sur. Baja ligeramente su magnitud alrededor de la marea baja, o cuando el viento disminuye. Una estratificación de flujos se mostró en 

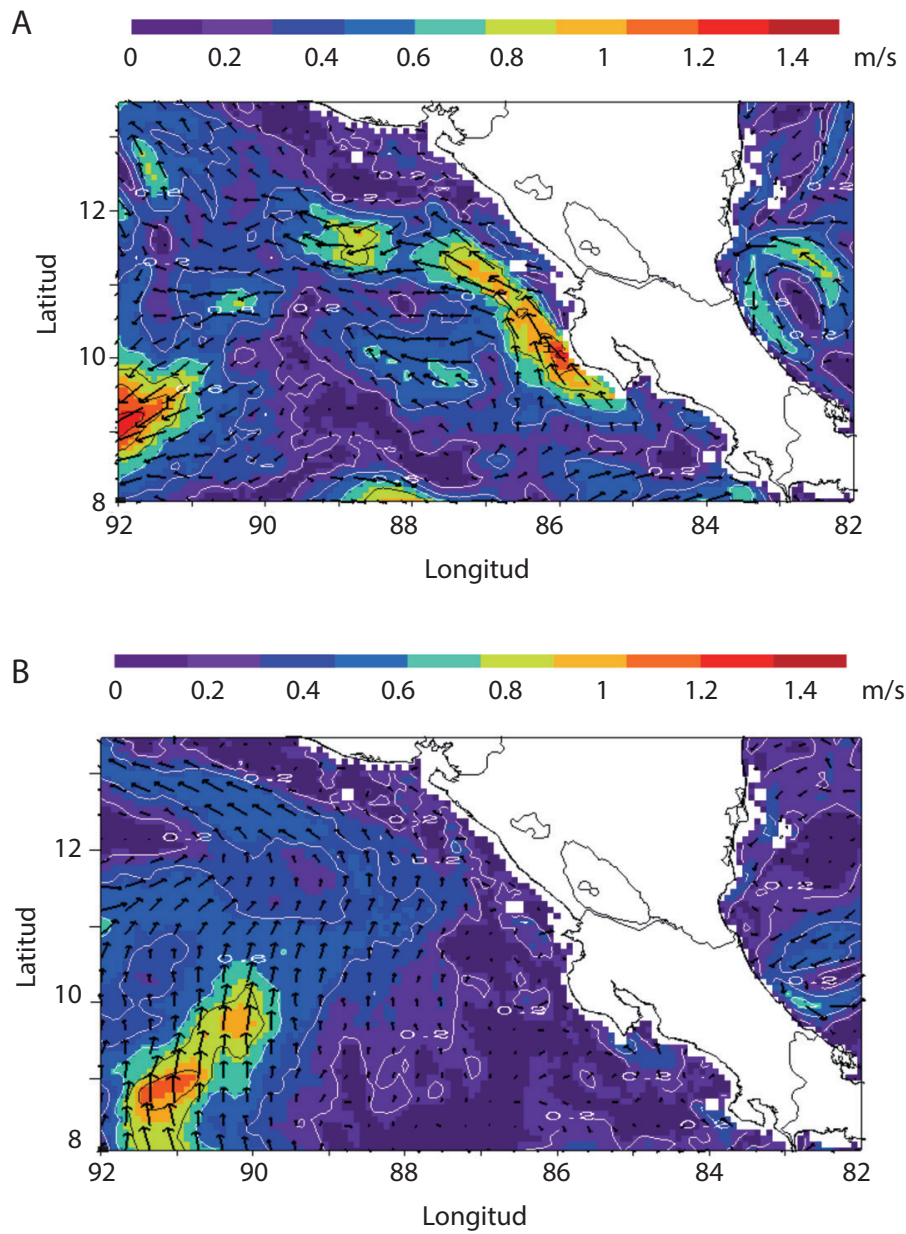

Fig. 17. Vectores y magnitudes de corrientes marinas superficiales frente al Pacífico de Costa Rica, resultados del modelo HYCOM (MIO-CIMAR) en A: 1 de marzo y B: 20 de junio.

Fig. 17. Surface marine currents, vector and magnitude off the Costa Rican Pacific, A: March 1 and B: June 20.

las corrientes en la mañana en Bahía Culebra, cuando la marea estaba subiendo (marea alta a las 08:29 hrs). Por la tarde el flujo fuerte fue en la superficie, cuando la marea estuvo bajando. Aunque en este sitio el flujo promedio también se mantuvo hacia el sur y sur-sureste todo día, la marea tiene alguna influencia en la circulación general de las corrientes marinas frente a esta región.

De acuerdo a los resultados de la campaña en Bahía de Santa Elena, las corrientes marinas ambientales que prevalecían en este momento (Fig. 17B), indicaban que no había un patrón claro y definido, como sí lo hubo en el marzo pasado. Aquí si se notó un cambio de dirección en el flujo de las corrientes en respuesta a los cambios de marea. Mientras la marea estaba bajando (marea baja a las 15:19 hrs), el flujo de agua era débil $(13 \mathrm{~cm} / \mathrm{s})$, y hacia afuera de la bahía con dirección noroeste (Fig. 11A), y cuando la marea estaba subiendo (marea alta a las 9:30 am), el flujo era más intenso por la superficie $(37 \mathrm{~cm} / \mathrm{s})$ hacia el sureste, hacia el interior de la bahía (Fig. 11B), coincidiendo con el incremento en la velocidad del viento. Pero también hubo estratificación en la dirección de las corrientes en la columna de agua, y más evidente durante la tarde del 20 
de junio. Conforme la velocidad del viento incrementó, el flujo en superficie ingresaba a la bahía (hacia el sureste), mientras que en el fondo la dirección era noroeste (saliendo de la bahía), una estratificación esperada en una bahía semi-cerrada.

\section{Condiciones regionales y locales para} la campaña de julio del 2012. La revisión de condiciones ambientales frente al Golfo de Nicoya (consultadas en la página MIO: www. miocimar.ucr.ac.cr), indicaban un viento oeste débil $(<2 \mathrm{~m} / \mathrm{s})$. Las corrientes marinas frente a esta región también eran débiles, sin un patrón definido, como se pudo revisar en el MIO para el 30 de julio del 2012. Bajo estas condiciones, y como esta bahía está dentro del Golfo de Nicoya, su circulación estuvo mas influenciada por la marea, la cual es una de las principales fuentes de la dinámica de las aguas en esta parte externa del golfo (Lizano \& Alfaro, 2004; Voorhis et al., 1983). El análisis de corrientes del ADCP evidencian la influencia de la marea en esta estación, aunque tienen un desfase respecto de la marea de Puntarenas, resultados que también se reportaron por Lizano \& Alfaro (2004). Fue clara una estratificación de flujos en la columna de agua, tanto en la magnitud de la velocidad de las corrientes, como en dirección, lo cual es una característica de la dinámica de esta bahía dentro de un golfo, con una gran influencia de mareas (Lizano \& Alfaro, 2004).

\section{AGRADECIMIENTOS}

El estudio se realizó dentro del marco de los siguientes proyectos de la Vicerrectoría de Investigación de la Universidad de Costa Rica: 808-B2-400 (apoyado por la Fundación Costa Rica x Siempre y la Agencia Alemana de Cooperación GiZ a través del Proyecto BIOMARC), 808-A9-180, 808-B0-040, 805B3-600 (apoyo del fondo de estímulo UCR), 805-A9-224 (apoyo del fondo de estímulo UCR), UCR.

\section{RESUMEN}

Se estudiaron las condiciones oceanográficas y meteorológicas en cuatro regiones relacionadas con parques nacionales en el Área de Conservación Guanacaste. Parámetros como temperatura, salinidad y oxígeno disuelto se estudiaron a través de la columna de agua en al menos un ciclo de marea en las bahías Tamarindo, Culebra, Santa Elena en el Pacífico Norte de Costa Rica, y en Bahía Ballena en la parte externa del Golfo de Nicoya. Al mismo tiempo se midieron corrientes marinas y viento local, de manera que se pudieran evaluar las fuerzas dinámicas en estos sitios. Se consultaron variables ambientales desde fuentes de datos operacionales alrededor de Costa Rica, como las corrientes marinas superficiales y el patrón de viento al frente de esta región, para conocer las condiciones prevalecientes y su relación con la dinámica de las variables medidas en cada sitio. Se encontró que cuando el viento sopla fuerte sobre esta zona, una condición típica de la época seca en el Pacífico Norte de Costa Rica, las corrientes marinas son intensas al frente de la Península de Nicoya. Esta es una característica coherente con las condiciones oceanográficas existentes cuando estos vientos se generan mar adentro al frente del Golfo de Papagayo. Estas fuerzas externas influencian la circulación de bahías abiertas sobre esta región, como en la Bahía de Tamarindo. El viento local y la marea son componentes que modulan la circulación local, especialmente en bahías muy cerradas como la Bahía de Santa Elena y Bahía Ballena. La estructura de la capa de agua en Bahía Ballena muestra una estratificación de corrientes, evidenciando una condición estuarina típica de la presencia de diferentes campos termohalinos dominados por las fuerzas de marea.

Palabras clave: dinámica atmosférica, temperatura del mar, oxígeno disuelto, circulación de estuarios, Área de Conservación Guanacaste.

\section{REFERENCIAS}

Alfaro, E.J., Cortés, J., Alvarado, J., Jiménez, C., Sánchez, C., Nivia, J., León, A., \& Ruiz, E. (2012). Clima y temperatura sub-superfical del mar en Bahía Culebra, Costa Rica. Revista de Biología Tropical,60(Suplemento2), 159-171.

Alfaro, E.J., \& Cortés, J. (2012). Atmospheric forcing of cool subsurface water events in Bahía Culebra, Costa Rica.Revista de Bilogía Tropical, 60(Suplemento2), 173-186.

Alfaro, E.J. (2002). Some characteristics of the annual precipitation cycle in Central America and their relationships with its surrounding tropical oceans. Tópicos Meteorológiocs y Oceanográficos, 9, 88-103.

Amador, J. A., Alfaro, E.J., Lizano, O. G., \& Magaña, V. (2006). Atmospheric forcing in the Eastern Tropical 
Pacific: A review. Progress in Oceanography, 69 101-142.

Badan-Dangon, A. (1998). Capter 11: Coastal circulation from the Galapagos to the Gulf of California. The Sea. Nueva York, NY: John Wiley.

Brenes, C. L., M. F. Lavín, . F. M., \& Mascarenhas Jr. A. S. (2008). Geostrophic circulation between the Costa Rica Dome and Central America. Deep-Sea Research $I$, doi:10.1016/j.dsr.2008.02.005.

Fiedler, P. C. (2002). The annual cycle and biological effects of the Costa Rica Dome. Deep-Sea Research, 49, 321-338.

Fonseca, A. C. (2006). CAPITULO XIV: Golfo de Papagayo: In: V. N. Muñoz \& M. A. Quesada (Eds.), Ambientes Marino Costeros de Costa Rica (pp. 185194). Comisión Interdisciplinaria Marino Costera de la Zona Económica Exclusiva de Costa Rica.

Kessler, W. S. (2006). The circulation of the eastern tropical Pacific: A review. Progress in Oceanography, 69, 181-217.

Lavín, M. F., Beier, E., Gómez-Valdés, J., Godínez, V.M., \& García, J. (2006). On the summer poleward coastal current off SW México. Geophysical Reseach Letters, 33, L02601, doi:10.1029/2005GL024686, 2006.
Lizano, O. G. (2007). Climatología del viento y oleaje frente a las costas de Costa Rica. Ciencia y Tecnología, 25(1-2), 43-56.

Lizano, O.G., \&Alfaro, E.J. (2004). Estructura de las corrientes marinas en el Golfo de Nicoya. Revista de Biología Tropical, 52(Suplemento 2), 74-94.

Magaña, V., Amador, J. A., \& Medina, S. (1999). The midsummer drought over Mexico and Central America. Journal of Climate, 12, 1577-1588.

Taylor, M.A., \& Alfaro, E.J. (2005). Climate of Central America and the Caribbean. In J.E. Oliver (Ed.), The Encyclopedia of World Climatology (pp. 183-189). Netherlands: Springer.

Voorhis, A. D., Epifanio, C. E., Don Maurer, Dittel, A. L., \& Vargas, J, A. (1983). The estuarine character of Gulf of Nicoya, an embayment on the Pacífic coast of Central America. Hydrobiologia, 99, 225-237.

Wyrtki, K. (1965). Surface currents of the eastern tropical Pacific ocean. Inter-American Tropical Tuna Commission Bulletin, 9, 271-304. 
